\title{
Characterization of Salvia Miltiorrhiza ethanol extract as an anti-osteoporotic agent
}

\author{
Yan Cui', Bidur Bhandary ${ }^{1}$, Anu Marahatta', Geum-Hwa Lee², Bo Li', Do-Sung Kim', Soo-Wan Chae', \\ Hyung-Ryong Kim ${ }^{2^{*}+}$ and Han-Jung Chae ${ }^{1 *^{*+}}$
}

\begin{abstract}
Background: Salvia miltiorrhiza (SM) has long been used as a traditional oriental medicine for cardiovascular disease. Accumulating evidence also indicates that SM has anti-osteoporotic effects. This study was conducted to examine the SM-induced anti-osteoporotic effect and its possible mechanisms with various doses of SM.

Methods: We studied Sprague-Dawley female rats aged 12 weeks, divided into six groups: sham-operated control (SHAM), OVX rats supplemented with SM (1,3,10 and $30 \mathrm{mg} / \mathrm{kg})$ orally for 8 weeks. At the end of the experiment, blood samples were collected and biochemistry analysis was performed. Specimens from both tibia and liver were processed for light microscopic examination. DEXA and $\mu-C T$ analyses of the tibia were also performed.

Results: SM treatment significantly ameliorated the decrease in BMD and trabecular bone mass according to DEXA and trabecular bone architecture analysis of trabecular bone structural parameters by $\mu$-CT scanning. In serum biochemical analysis, SM decreased the released TRAP-5b, an osteoclast activation marker and oxidative stress parameters including MDA and NO induced by OVX.

Conclusions: The preventive effect of SM was presumably due to its anti-oxidative stress partly via modulation of osteoclast maturation and number. In current study, SM appears to be a promising osteoporosis therapeutic natural product.
\end{abstract}

Keywords: Salvia miltiorrhiza, OVX, BMD, morphometry, oxidative stress

\section{Backgrounds}

Osteoporosis is a multifactorial skeletal disease that is characterized by compromised bone strength predisposing a person to an increased risk of fracture [1]. Longterm administration of currently prevalent medications may lead to an increased risk of severe side effects like cancer [2]. Salvia miltiorrhiza (SM) has long been used as a traditional oriental medicine for cardiovascular disease. Accumulating evidence also indicates that SM has anti-osteoporotic effects. The dried root of Salvia miltiorrhiza Bunge (Labiatae) is also named Danshen or

\footnotetext{
* Correspondence: hrkimdp@wonkwang.ac.kr; hjchae@jbnu.ac.kr

† Contributed equally

'Department of Pharmacology and Institute of Cardiovascular Research, School of Medicine, Chonbuk National University, Jeonju, Chonbuk, South Korea

${ }^{2}$ Department of Dental Pharmacology and Wonkwang Dental Research Institute, School of Dentistry, Wonkwang University, Iksan, Chonbuk, South Korea

Full list of author information is available at the end of the article
}

Tanshen. The herb is mainly produced in Anhui, Shanxi, Hebei, Shuan, and Jiangsu provinces in China [3]. Among the chemical constituents of Danshen, there are tanshinone I, tanshinone IIA, tanshinone IIB, cryptotanshinone, tanshindiol C, 15,16-dihydrotanshinone I, isotanshinone I, isotanshinone II and other tanshinones [4]. Several biological activities have been detected for the major tanshinones through in vivo and/or in vitro tests, such as antioxidant, anti-inflammatory, antimicrobial, anti-menopausal syndrome, anti-ischemic, and antineoplastic activities $[3,5,6]$. The inhibitory effect of tanshinone IIA on osteoclast differentiation and bone resorption was also observed [7]. Consistently, SM significantly increases the blood estrogen level in ovariectomized (OVX) rats, suggesting that SM might help prevent bone resorption in this osteoporosis model $[7,8]$. These results were also related with a study suggesting that SM has a positive effect on promoting angiogenesis [9]. Wong et al. also showed that SM 
extract increased bone formation through the combined actions of increasing angiogenesis, increasing osteoblastic activity and decreasing osteoclastic activity $[10,11]$. Our previous study revealed that aqueous extract of SM effectively prevents the development of bone loss induced by OVX in rats [12]. However, a detailed characterization of the effect of SM has not been elucidated yet.

The aim of the current study is to clarify the antiosteoporotic effect of SM at various doses. This study was performed in OVX rats by observing the changes in biochemistry data, bone mineral density (BMD), trabecular bone structural morphometric traits and histological characteristics.

\section{Methods \\ Materials}

The dried root slices of SM were acquired from Hansol Oriental Medical (Gimje, Korea). 1800 g of SM powder were prepared from dried root slices of SM that were cut into small pieces and extracted with $100 \%$ ethanol at $78^{\circ} \mathrm{C}$ for $3 \mathrm{hr}$ in triplicate. The extract was filtered, evaporated on a rotary vacuum evaporator at $50^{\circ} \mathrm{C}$ and freeze-dried to yield $26.52 \mathrm{~g}$ of SM extract. $106.56 \mu \mathrm{g}$ of tanshinone IIA/10 mg of SM extracts (1.07\%) and $109.655 \mu \mathrm{g}$ of crytotanshinone/10 mg of SM extracts (1.10\%) was verified by high performance liquid chromatography (HPLC).

The chemical products used in the experiment include: methanol and acetic acid of HPLC grade (Merck, Germany). Tanshinone IIA and cryptotanshinone standards were purchased from Sigma Company (USA). Rompun (1 ml of Rompun contains $23.32 \mathrm{mg}$ of Xylazine hydrochloride) was purchased from Bayer Korea (Ansan, Korea) and Ketamine was acquired from Yuhan (Seoul, Korea). Estradiol Depot was obtained from Jenapharm (KG, Germany).

\section{Experimental Animals}

Twelve-week-old female Sprague-Dawley rats, weighing 230-270 g, were purchased from Damul Science Co (Daejeon, Korea), allowed to acclimate for 7 days, and kept another 7 days for a baseline period before the start of the experiment. The rats were maintained at a constant temperature $\left(25 \pm 2^{\circ} \mathrm{C}\right)$ and humidity $(55 \% \pm$ $5 \%)$, with a cycle of 12 hours light and 12 hours darkness. They were housed individually in standard cages and were provided with ad libitum tap water and a commercial standard diet containing $1.2 \%$ calcium and $0.8 \%$ phosphorus. All procedures using animals were carried out in accordance with the guidelines presented in the "Principles for the Care and Use of Animals in the Field of Physiological Sciences", published by the Physiological Society of Korea. The study protocol was approved by an ethics committee in Chonbuk National University (Jeonju, Korea). Experiment animals were allocated to sham-operated (Sham), OVX-control (OVX), and 1, 3, 10 and $30 \mathrm{mg} / \mathrm{kg} \mathrm{SM}$ treated (1SM, 3SM, 10SM and 30SM) ovariectomized groups for a total of 6 groups $(\mathrm{N}=10 /$ each group). Rats in the sham-operated group underwent a sham operation, i.e., only the skin incision was made. Briefly, the operations were performed by exteriorizing the ovaries after the baseline period at week 0 ; the other rats were ovariectomized. Rats were operated on while under anesthesia by a mixture of Ketamine $(35 \mathrm{mg} / \mathrm{kg}$ ) and Xylazine $(10 \mathrm{mg} /$ $\mathrm{kg}$ ) administrated intraperitoneally. Success of OVX was confirmed at necropsy by retrospectively inspecting atrophy of the uterine horns [13,14]. After a 1-week healing period, rats in the drug-treated ovariectomized groups were orally treated with a series dosage of SM once daily for 8 weeks and Sham and OVX groups were orally treated with volume-matched vehicles before sampling. The doses and durations of SM treatment were predetermined on the basis of preliminary studies [15]. The body weight of each rat was measured weekly, and the dosage of drug or vehicle administered was calculated based on the most recent body weight measurement. After 8 weeks of drug administration, the experimental rats were fasted overnight; the next morning, rats were anesthetized and blood was sampled from the abdominal aorta. Serum was isolated from the blood samples by centrifugation at $3000 \times \mathrm{g}, 4^{\circ} \mathrm{C}$, for $5 \mathrm{~min}$ and stored at $-70^{\circ} \mathrm{C}$ prior to biochemical measurement. After the blood sample was collected, the rats were bled to death, and the liver and tibiae were excised. The liver and left tibia (defleshed) of each animal were fixed with fixative and used for further histomorphometric analysis, while the right tibia was freed of all soft tissue and wrapped in a layer of PARAFILM (Menasha co., U.S.A), apart from $5 \mathrm{~mm}$ of its proximal end, and fixed into a $15 \mathrm{ml}$ BD Falcon Tube (BD Co., Franklin Lakes, NJ USA) and then soaked in fixative. The tube cap was tightened before performing a $\mu$-CT scan to measure the microstructural parameters. The right femurs were subjected to DEXA measurement for BMD and bone mineral content (BMC).

\section{Bone $\mu$-CT Scanning}

To assess bone loss, rats right tibiae (defleshed) were ex vivo scanned at the end of drug treatment. A 6-mm $\mu$ CT scan $(70 \mathrm{kV}, 85 \mathrm{lA}, 1,000$ projections per 180 degrees, $350 \mathrm{~ms}$ integration time) with an isotropic resolution of $18 \mu \mathrm{m}$ was made of the proximal tibia using an in vivo $\mu$-CT scanner (SkyScan-1076 in vivo CT-scanner; SkyScan, Belgium) The CT scanner was calibrated, and a beam hardening correction algorithm was applied to all scans. One CT scan took 35 minutes. In this study, 
the reproducibility of all structural parameters was high, with a coefficient of variation of about $1 \%$.

From the stack of cross-section images, a volume of interest (VOI) containing only cancellous bone was extracted for morphometric analysis (CT Analyzer V 1.11.0.0, Skyscan, Kontich, Belgium). The VOI started at a distance of $1 \mathrm{~mm}$ from the lower end of the growth plate and extended distally for 110 cross sections $(2 \mathrm{~mm}$ in height). For morphometric analysis, the following structural parameters were calculated over each VOI of cancellous bone by "3D analysis" (using CT Analyzer software): bone volume fraction (BV/TV), connectivity density (Conn.D), trabecular thickness (Tb.Th), direct trabecular separation (Tb.Sp), trabecular number (Tb.N), trabecular pattern factor (Tb.Pf), BMD, and structure model index (SMI). SMI indicates whether the trabeculae are more rod-like $(\mathrm{SMI}=3)$ or more plate-like (SMI $=0$ ); Lower Tb.Pf signifies better connected trabecular lattices while higher Tb.Pf means a more disconnected trabecular structure; Conn.D was obtained by calculating the connectivity of the trabecular network and normalized by dividing the connectivity by bone volume (BV/ TV) [14].

The cortical area of the diaphyseal region of the tibia was also calculated using CT Analyzer software. The cut level for measurement of the cortical area was defined at a distance of $8 \mathrm{~mm}$ from the lower end of the growth plate. The cortical area (Ct.Ar), and cortical thickness (Ct.Th) were analyzed by "Individual 2D object analysis" in CT Analyzer software, and cortical thickness was calculated by the formula $\mathrm{Ct}$. Th $=1 / 2 \times \mathrm{BS} / \mathrm{BV}$. The above formula is defined as: area of a ring = thickness of ring $\times$ length of middle line $=$ thickness $\times$ (outer circumference + inner circumference) $/ 2$ [14].

The average attenuation coefficient of the trabecular bone tissue was determined for all measurements using a protocol provided by the manufacturer of the $\mu$-CT scanner. With this protocol, the gray levels of voxels near the trabecular surfaces are not included to ensure that the measurements are not affected by partial volume effects.

\section{DEXA Measurement}

All DEXA measurements were performed by the same investigator (Y. C) using the Norland pDEXA Sabre (Fort Atkinson, WI, USA) equipped with Sabre Research software (v3.6). The interassay coefficient of variation (CV) for BMD and BMC was $1.7 \%$. The scanner was calibrated daily to a dual-material standard according to the manufacturer's recommendations, and the scanner performance was controlled by the quality assurance protocol of our laboratory. The right femurs were scanned using DEXA to determine BMC and BMD. Ex vivo measurements of the right distal femur were performed on excised bones positioned onto a 3-mmthick cotton piece on the bottom (thickness $1 \mathrm{~mm}$ ) of a 10-cm diameter culture dish at a constant location on the scan table, and measured by DEXA using a special collimator ( $0.8 \mathrm{~mm}$ diameter); the scan length was $5 \mathrm{~cm}$, the scan width $2 \mathrm{~cm}$ and the scan speed $10 \mathrm{~mm} / \mathrm{s}$ with a resolution of $0.2 \mathrm{~mm} \times 0.2 \mathrm{~mm}$ [16-18]. The deltoid tuberosity was faced upward to avoid an irregular projecting shape; the starting point of the scan was above the distal condyle of the femur and the end point was proximal to the femoral end so that the scanner arm moved along the long axis of the femoral shaft allowing evaluation of femur length. The baseline point was located on the cotton piece $[16,19]$.

\section{Liver Histological Examination}

Liver specimens were fixed in $10 \%$ buffered neutral paraformaldehyde solution, processed and embedded in paraffin. Thin paraffin sections (5- $\mu \mathrm{m}$ thick) were stained by hematoxylin and eosin ( $\mathrm{H} \& \mathrm{E})$. The numbers of mononuclear cells were determined/10 HPF.

\section{Bone Histomorphometric Analysis}

Left tibiae were decalcified in 5\% formic acid (in distilled water) solution for 1 week, dehydrated with methanol, and embedded in paraffin. The paraffin sections were deparaffinized and stained $(\mathrm{H} \& \mathrm{E})$. Sections with the widest marrow cavity near the growth plate of the metaphysis of tibiae were selected for further histological processing and histomorphometric measurements.

Histomorphometrical measurements were made using an Optiphot 2 microscope connected to a RGB camera and a personal computer (software: Lucia G 4.51, Laboratory Imaging), with final magnifications of $30 \times$ and $400 \times$. The number of osteoclasts (Oc.N) was determined/10 HPF.

\section{BALP Enzyme Assay}

Rat bone alkaline phosphatase (BALP) enzyme-linked immunosorbent assay (ELISA) kit was provided by Cusabio Biotech Co., LTD. (Wuhan, China). Rat BALP was also measured using ELISA from R \& D Systems (Minneapolis, MN, USA).

\section{TRAP Enzyme Assay}

Rat TRAP-5b EIA Kit was obtained from KAMIYA BIOMEDICAL Company (Seattle, WA, USA). Rat TRAP-5b was also measured by ELISA (R \& D Systems, Minneapolis, MN, USA).

\section{Plasma Peroxide Assay}

The plasma malondialdehyde (MDA) levels were determined according to the method of Draper and Hadley 
(1990) [20], based on the reaction of MDA with thiobarbituric acid. Measurement was conducted using the lipid peroxidation assay kit (Calbiochem). The absorbance at $586 \mathrm{~nm}$ was measured using an ELISA microplate reader.

\section{Plasma Nitrate Assay}

Plasma nitrate levels were measured according to the method of Bories and Bories (1995) [21]. Total serum nitric oxide (NO) was calculated based on the enzymatic conversion of nitrate to nitrite by nitrate reductase, using a commercial kit (Total Nitric Oxide and Nitrate/ Nitrite Parameter Assay Kit, R\&D SYSTEMS, Minneapolis, MN, USA).

\section{Biochemical Analysis of Serum Parameters}

Serum content of calcium, inorganic phosphorus (IP), ALP, triiodothyronine $\left(\mathrm{T}_{3}\right)$, thyroxine $\left(\mathrm{T}_{4}\right)$, osteocalcin, estradiol, intact PHT and calcitonin were determined using standard laboratory techniques. Serum levels of free $\mathrm{T}_{4}$, free $\mathrm{T}_{3}$, intact $\mathrm{PTH}$, and estradiol were measured with free $\mathrm{T}_{3}$, free $\mathrm{T}_{4}$, Elecys PTH, and Estradiol $\alpha$ kits (Roche, Germany), respectively, using Modular Analytics E170 (Roche, Germany) in the electrochemiluminescence immunoassay method. Serum calcium and IP were measured with related kits (Roche, Germany) using Modular Analytics PE (Roche, Germany) in the colorimetric and phosphomolybdate \& ultraviolet spectrophotometric methods, respectively. Serum ALP activity was measured with ALP kit (Roche, Germany) using Modular Analytics PE (Roche, Germany) with colorimetry with PNPP. Calcitonin was measured with Liaison calcitonin $\alpha$-Gen kit (Diasorin, USA) by the chemiluminescent immunoassay method.

\section{Statistical Analysis}

Data are expressed as means \pm SD. Statistical significance for data was determined using one-way analysis of variance (ANOVA) with post-hoc test, and significance was calculated by LSD (least significant difference) multiple range-test to find inter-group significance. The level of significance was accepted as $p<0.05$.

\section{Results}

\section{Preparation of SM extracts}

In the pure components of SM, tanshinone I, tanshinone IIA, tanshinone IIB, cryptotanshinone, tanshindiol C, 15,16-dihydrotanshinone I, isotanshinone I, isotanshinone II and other tanshinones are included [4]. Among the tanshinone compounds, tanshinone IIA and cryptotanshinone were selected as active and quality control compounds in this study. Calibration curves of the two compounds were constructed by measuring different concentrations. Good linearity was observed for tanshinone IIA $\left(\mathrm{r}^{2}=0.9911\right)$ and cryptotanshinone $\left(\mathrm{r}^{2}=\right.$ $0.9921)$. The regression equations for $t$ anshinone IIA and cryptotanshinone were $y=59467 x+296829$ and $y$ $=62354 \mathrm{x}-109248$, respectively (data not shown). The typical HPLC-UV profiles are illustrated in Additional file 1. The HPLC condition has been also described in Additional file 2. Good separation was achieved within $25 \mathrm{~min}$. The retention times for cryptotanshinone and Tanshinone IIA were 14.8 and $21.6 \mathrm{~min}$. The content of tanshinone IIA and cryptotanshinone in Salvia Miltiorrhiza was determined from the corresponding regression equation. Tanshinone IIA content was $106.56 \mu \mathrm{g} / 10 \mathrm{mg}$ of SM extract (1.07\%) whereas cryptotanshinone content was $109.655 \mu \mathrm{g} / 10 \mathrm{mg}$ of $S M$ extract $(1.10 \%)$.

\section{Body Weight Changes}

As time passed from 2 to 8 weeks after OVX, the average body weight growth in the OVX groups was significantly greater than that in the Sham group $(p<$ $0.05 \sim 0.001$ ), but administration of SM did not affect the body weight growth pattern (Additional file 3).

\section{BMD and BMC}

In DEXA ex vivo measurement, the aBMD and aBMC of right distal femora were significantly decreased by $38 \%$, respectively, by OVX $(p<0.001)$. SM administration provided some degree of safety in a dose-dependent manner, but only high-dosage SM $(30 \mathrm{mg} / \mathrm{kg}$ body weight/day) treatment significantly prevented aBMD and aBMC reduction by $33 \%$, respectively $(p<0.05)$ (Figure $1 \mathrm{~A}, \mathrm{~B})$. In $\mu$-CT ex vivo measurement, the vBMD of proximal tibiae was significantly reduced by $74 \%$ ( $p<$ 0.001 ), and SM treatment resulted in the same pattern as in DEXA measurement, i.e., the vBMD decrease was prevented by $22 \%$ only in $30 \mathrm{SM}$ rats $(p<0.05)$ (Figure $1 C)$. This study showed the coronal images of rat medial-proximal tibia by $\mu-\mathrm{CT}$ (Figure $2 \mathrm{~A}$ ) and 3D images $\mu$-CT (Figure $2 \mathrm{~B}$ ) with the taken by SM dose-dependent prevention about bone loss in OVX rats.

\section{$\mu-C T$ Evaluation}

To examine the effect of SM on BMD, coronal image of proximal-medial tibia was taken ex vivo by $\mu$-CT. A. Additional file 4 showed setting conditions for the $\mu$ CT. Table 1 showed that OVX induced significant changes in all trabecular microstructural parameters in the proximal tibial metaphysis measured by $\mu-C T$. Compared with Sham rats, OVX significantly reduced bone volume fraction $(\mathrm{BV} / \mathrm{TV})$, by $87 \%(p<0.001)$, trabecular thickness (Tb.Th) by $14 \%$ ( $p<0.001)$, trabecular number (Tb.N) by $85 \%(p<0.001)$ and connectivity density (Conn.D) by $91 \%(p<0.001)$, and increased trabecular separation (Tb.Sp) by $320 \%(p<0.001)$. Other microstructural parameters such as SMI and trabecular bone 

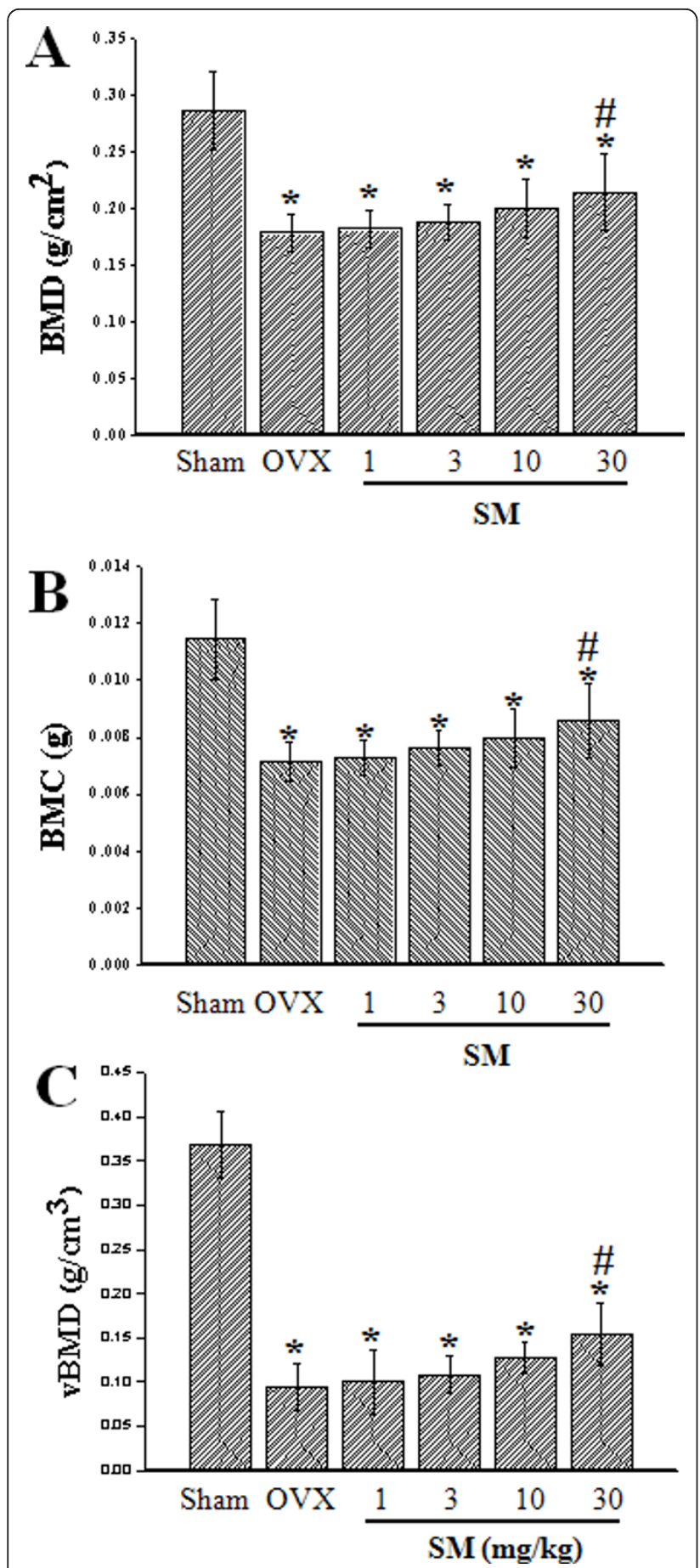

Figure 1 The Quantification of BMD and BMC in SM-treated rats. $\mathrm{aBMD}(\mathrm{A})$ and $\mathrm{aBMC}(\mathrm{B})$ of right distal femur ex vivo measured by DEXA, and $V B M D(C)$ of right proximal tibia ex vivo measured $\mu$ $C T$. OVX significantly decreased $\mathrm{aBMD}$, by $\mathrm{aBMC}$ and $\mathrm{VBMD}$, respectively. Although SM treatment tended to have a dosedependent preventive effect, only treatment with $30 \mathrm{mg} / \mathrm{kg}$ body weight/day of SM significantly prevented BMD and BMC decrease induced by OVX. Note: ${ }^{*} p<0.001$ vs Sham group; ${ }^{\#} p<0.05$ vs OVX group pattern (Tb.Pf) were also significantly different $(p<$ 0.001). SM treatment also showed some tendency for dose-dependent safety effects but only the maximum SM treatment of $30 \mathrm{mg} / \mathrm{kg}$ had a significant preventive effect, attenuating reduction of BV/TV by $24 \%(p<$ $0.05)$, Tb.Th by $65 \%(p<0.05)$, Tb.N by $23 \%(p<0.05)$ and Conn.D by $12 \%(p<0.05)$, while preventing increase of Tb.Sp by $43 \%(p<0.05)$, SMI by $30 \%(p<$ $0.05)$ and Tb.Pf by $28 \%(p<0.05)$. Ct. Ar and Ct. Th measured by $\mu$-CT were also summarized in the Table 1. OVX did not affect the cortical area and thickness of tibial diaphysis.

\section{Bone Histomorphometric Parameters}

As shown in Table 2 and Figure 3, the histomorphometric parameters were analogous to the $\mu$-CT observations of trabecular morphology: OVX significantly reduced BV/TV by $82 \%(p<0.001)$, Tb.Th by $58 \%(p<$ $0.001)$, Tb.N by $64 \%(p<0.001)$, and increased Tb.Sp by $604 \%(p<0.001)$. SM treatment also tended to have a dose-dependent preventive effect at the experimental dosages, but only treatment with the maximum of 30 $\mathrm{mg} / \mathrm{kg}$ body weight $/ \mathrm{kg}$ of SM showed significance, attenuating reduction of BV/TV by $19 \%(p<0.05)$, Tb. Th by $57 \%(p<0.05)$, and Tb.N by $65 \%(p<0.05)$, while preventing the increase of Tb.Sp by $69 \%(p<$ $0.05)$. OVX also induced a significant increase in Oc.N $(p<0.001$ vs. Sham), and SM treatment attenuated the Oc.N increase only in the $30 \mathrm{SM}$ group ( $p<0.05$ vs. OVX).

\section{Liver Histomorphometry}

As shown in Figure 4 and Table 3, OVX aggravated mononuclear cellular infiltration in the portal area of the liver ( $p<0.001$ vs. Sham rats) and SM treatment significantly ameliorated mononuclear cellular infiltration only at $30 \mathrm{mg} / \mathrm{kg}$ body weight/day ( $p<0.05 \mathrm{vs}$. OVX rats).

\section{Quantification of Serum bone turnover markers}

As shown in Figure 5A, serum BALP as a bone formation marker [22] was significantly increased in OVX rats $(p<0.05)$, while drug treatment did not affect the increase.

TRAP $5 \mathrm{~b}$ in serum is proposed to be a marker for osteoclasts [23]. As shown in Figure 5B, serum TRAP 5 b was significantly increased in OVX rats compared with Sham group $(p<0.05)$ but was significantly attenuated in 30SM group $(p<0.05)$, consistent with exchange in osteoclast number measured by histological assessment (Table 2) and indicating increased bone resorption. In order to understand the mechanism of SM on bone resportion parameter, malondialdehyde (MDA) 
B

A
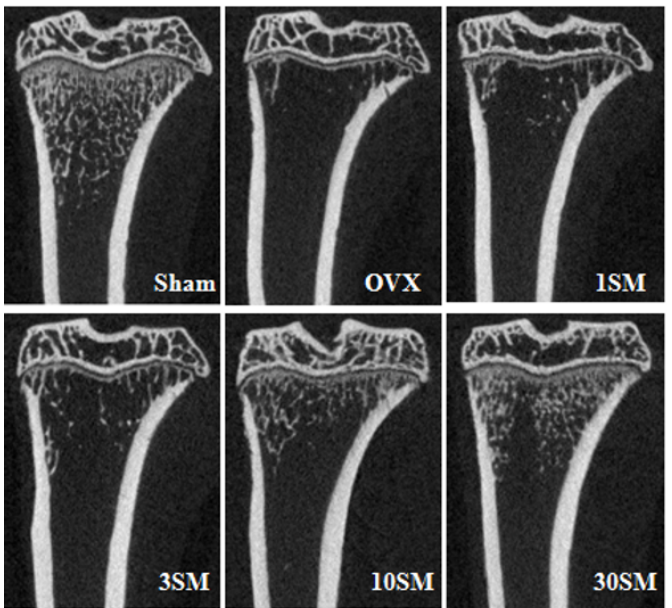
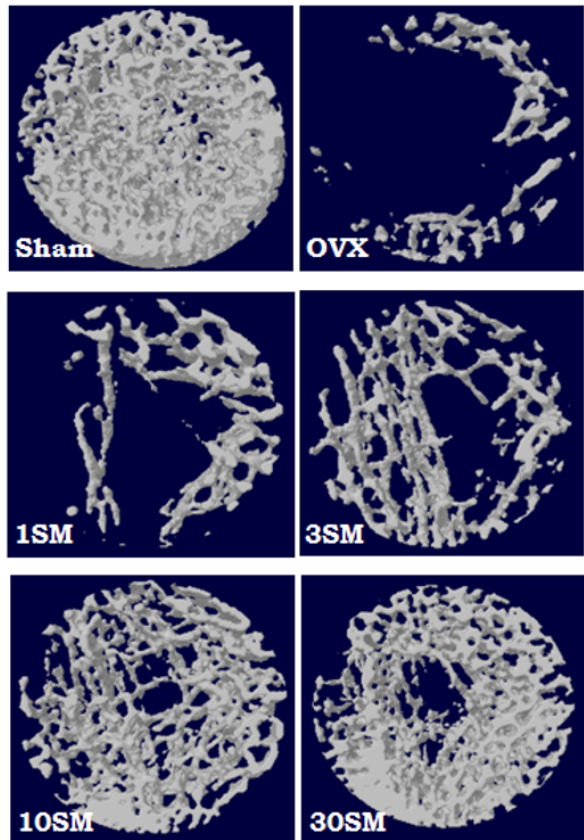

Figure 2 The image analysis of rat medial-proximal tibia in SM-treated rats. A, Coronal images of rat medial-proximal tibia were shown by $\mu-C T$. B, 3D images taken by $\mu-C T$.

Table 1 Trabecular microstructural properties of the right tibial metaphysis and cortical geometric properties evaluated ex vivo using micro-CT.

\begin{tabular}{|c|c|c|c|c|c|c|}
\hline Parameters & $\begin{array}{l}\text { Sham } \\
n=10\end{array}$ & $\begin{array}{c}\text { OVX } \\
n=10\end{array}$ & $\begin{array}{c}\text { SM1 } \\
\mathrm{n}=7\end{array}$ & $\begin{array}{c}\mathrm{SM} 3 \\
\mathrm{n}=8\end{array}$ & $\begin{array}{c}\text { SM10 } \\
\mathrm{n}=10\end{array}$ & $\begin{array}{l}\text { SM30 } \\
\mathrm{n}=9\end{array}$ \\
\hline BV/TV (\%) & $30.06 \pm 4.57$ & $3.90 \pm 1.61 *$ & $4.56 \pm 2.44 *$ & $4.92 \pm 1.84$ * & $6.22 \pm 1.78 *$ & $8.05 \pm 3.32 *$, \# \\
\hline Tb.Th $(\mu \mathrm{m})$ & $112.76 \pm 7.95$ & $96.51 \pm 4.99 *$ & $101.03 \pm 6.58 *$ & $100.06 \pm 7.42 *$ & $99.53 \pm 5.92 *$ & $107.03 \pm 5.02 *$, \# \\
\hline Tb.N (1/mm) & $2.67 \pm 0.40$ & $0.40 \pm 0.16 *$ & $0.45 \pm 0.23 *$ & $0.50 \pm 0.19 *$ & $0.63 \pm 0.19 *$ & $0.93 \pm 0.28 *$, \# \\
\hline Tb.Sp (mm) & $0.25 \pm 0.08$ & $1.05 \pm 0.13 *$ & $1.05 \pm 0.26 *$ & $1.03 \pm 0.20 *$ & $0.93 \pm 0.21 *$ & $0.71 \pm 0.10 *$ \# \\
\hline Conn.D $\left(1 / \mathrm{mm}^{3}\right)$ & $97.53 \pm 12.90$ & $8.95 \pm 3.67 *$ & $9.24 \pm 4.90 *$ & $10.59 \pm 3.22 *$ & $14.24 \pm 6.62 *$ & $19.37 \pm 6.344^{*}, \#$ \\
\hline Tb.Pf $(1 / \mathrm{mm})$ & $4.25 \pm 2.29$ & $20.35 \pm 2.98 *$ & $19.50 \pm 2.26 *$ & $18.98 \pm 1.89 *$ & $18.48 \pm 2.62 *$ & $15.79 \pm 3.05 *$ \\
\hline SMI (1) & $1.46 \pm 0.25$ & $2.69 \pm 0.10 *$ & $2.64 \pm 0.12 *$ & $2.61 \pm 0.14 *$ & $2.59 \pm 0.16 *$ & $2.32 \pm 0.26 *$ \# \\
\hline Ct.Ar $\left(\mathrm{mm}^{2}\right)$ & $5.38 \pm 0.22$ & $5.41 \pm 0.45$ & $5.98 \pm 0.34$ & $5.67 \pm 0.43$ & $5.58 \pm 0.34$ & $5.29 \pm 0.31$ \\
\hline Ct.Th (mm) & $29.65 \pm 0.13$ & $29.63 \pm 0.09$ & $29.65 \pm 0.07$ & $29.54 \pm 0.10$ & $29.62 \pm 0.06$ & $29.66 \pm 0.08$ \\
\hline
\end{tabular}

Note: Results are presented as the means \pm SD. ${ }^{*} p<0.001$ vs. Sham group: ${ }^{*} p<0.05$ vs. OVX group. BV/TV, bone volume fraction; Conn.D, connectivity density; SMI, structure model index; Tb.Th, trabecular thickness; Tb.N, trabecular number; Tb.Sp, trabecular separation; Tb.Pf, Trabecular bone pattern factor; Ct.Ar, cortical area; Ct.Th, cortical thickness. Rat groups include sham-operated (Sham), OVX-control (OVX), and 1, 3, 10 and $30 \mathrm{mg} / \mathrm{kg}$ SM treated (1SM, 3SM, 10SM AND 30SM) ovariectomized groups for a total of 6 groups ( $N=7 \sim 10 /$ each group).

Table 2 Histomorphometric parameters of trabecular bone from metaphysis of tibiae evaluated by histochemical analysis.

\begin{tabular}{ccccccc}
\hline Parameters & $\begin{array}{c}\text { Sham } \\
\mathbf{n}=\mathbf{7}\end{array}$ & $\begin{array}{c}\text { OVX } \\
\mathbf{n}=\mathbf{7}\end{array}$ & $\begin{array}{c}\text { 1SM } \\
\mathbf{n = 7}\end{array}$ & $\begin{array}{c}\text { 3SM } \\
\mathbf{n}=\mathbf{7}\end{array}$ & $\begin{array}{c}\text { 10SM } \\
\mathbf{n}=\mathbf{7}\end{array}$ & $\begin{array}{c}\text { 30SM } \\
\mathbf{n}=\mathbf{7}\end{array}$ \\
\hline BV/TV (\%) & $55.44 \pm 7.10$ & $10.24 \pm 3.68^{*}$ & $11.51 \pm 2.27$ & $11.61 \pm 2.27$ & $15.19 \pm 1.61$ & $18.70 \pm 1.38^{\#}$ \\
Tb.Th $(\mathbf{m c m})$ & $35.14 \pm 5.17$ & $14.86 \pm 5.54^{*}$ & $15.72 \pm 3.33$ & $16.37 \pm 3.03$ & $21.32 \pm 3.71$ & $26.37 \pm 2.499^{\#}$ \\
Tb.N (1/mm) & $15.87 \pm 1.59$ & $5.73 \pm 2.03 *$ & $6.06 \pm 1.99$ & $6.39 \pm 2.00$ & $8.88 \pm 2.61$ & $12.35 \pm 2.67^{\#}$ \\
Tb.Sp (mcm) & $28.38 \pm 5.80$ & $199.70 \pm 3.48 *$ & $180.27 \pm 3.46$ & $176.26 \pm 2.18$ & $98.75 \pm 7.21$ & $81.67 \pm 6.38^{\#}$ \\
Oc.N/10 HPF & $5 \pm 1.41$ & $17 \pm 2.40^{*}$ & $16 \pm 2.39$ & $17 \pm 2.20$ & $13 \pm 2.26$ & $10 \pm 1.41^{\#}$ \\
\hline
\end{tabular}

Note: Results are presented as the means \pm SD. ${ }^{*} p<0.001$ vs. Sham group; ${ }^{*} p<0.05$ vs. OVX group. BV/TV, bone volume fraction; Tb.Th, trabecular thickness; Tb. $\mathrm{N}$, trabecular number; Tb.Sp, trabecular separation; Oc.N, osteoclast number. Rat groups as defined above ( $\mathrm{N}=7 / \mathrm{each}$ group). 


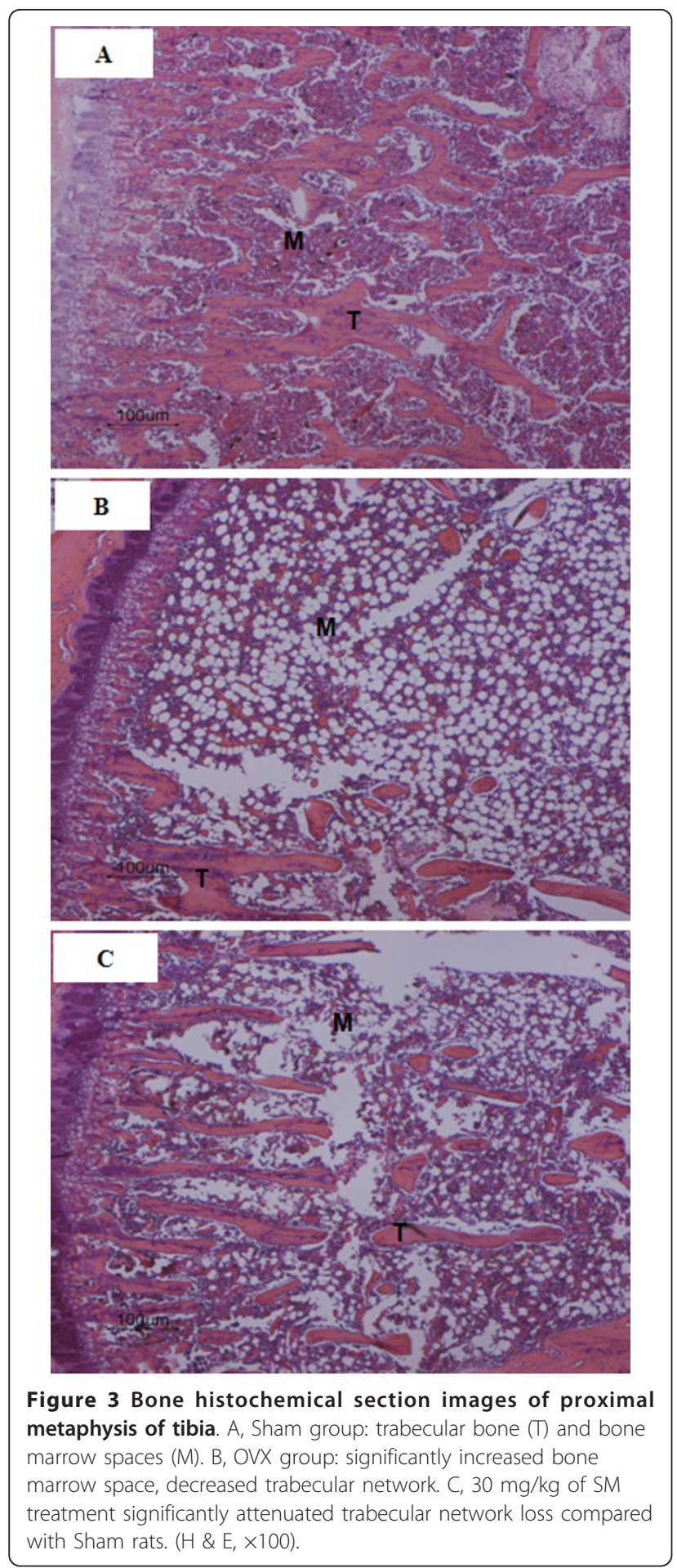

and nitric oxide (NO) were measured. OVX significantly increased serum MDA levels ( $p<0.001$ vs. Sham rats), meaning the induction of lipid peroxydation in OVX rats (Figure 5C). SM treatment, especially at the two groups, 10 and 30SM, significantly attenuated the MDA increase induced by $\operatorname{OVX}(p<0.001$ and $p<0.01$, respectively). Figure 5D showed that OVX significantly increased total serum nitrate, metabolite of NO $(p<$ 0.001 vs. Sham rats), and in $10 \mathrm{SM}$ and $30 \mathrm{SM}$ rats, SM treatment significantly prevented the nitrate increase induced by OVX ( $p<0.001$ vs. Sham rats).

\section{Serum Biochemical Levels}

Serum calcitonin and intact PTH levels were not significantly different among experimental groups (data not shown). As shown in Table 4, serum calcium and IP levels and free $\mathrm{T}_{3}$ were not significantly different among experimental groups, while OVX significantly decreased estradiol $(p<0.01)$ but the SM did not affect the decrease of estradiol. Free T4 was significantly increased in OVX rats $(p<0.001)$ and the increase was significantly attenuated in 30SM rats $(p<0.001)$. OVX significantly increased serum osteocalcin and ALP activity ( $p$ $<0.001$ and 0.05 , respectively) and SM treatment did not affect the increase.

\section{Discussion}

OVX induced significant trabecular bone loss due to estrogen deficiency and subsequent increased bone turnover. SM at $30 \mathrm{mg} / \mathrm{kg}$ body weight/day dosage significantly attenuated trabecular bone loss and BMD decrease induced by OVX. SM can contribute to bone balance probably through preventing an increase in osteoclast number by decreasing osteoclast maturation.

$\mathrm{SM}$ is a potential anti-osteoporotic natural product. For several decades, SM has been widely used for the treatment of various microcirculatory disturbancerelated diseases, such as cardiovascular disease, cerebrovascular disease, liver dysfunction, renal deficiency and diabetic vascular complications [4]. SM extract is also reported to increase bone formation through the combined actions of increased angiogenesis, increased osteoblastic activity and decreased osteoclastic activity [10]. In the current study, treatment with $30 \mathrm{mg} / \mathrm{kg}$ of ethanol extracts of SM significantly attenuated the dramatic decrease in BMD and deterioration in trabecular bone architecture (Table 1 \&2).

SM treatment also significantly prevented increases in serum nitrate and peroxide levels and ameliorated the increase in mononuclear cellular infiltration in the portal area of the liver (Table 3). In the current study, histological examination of the liver of the SM treated rats showed the regulatory effect of mononuclear cellular infiltration (Figure 4). Previous studies have showed that OVX condition induces liver inflammation $[24,25]$. The estrogen-induced prevention effect against bone loss may involve suppression of inflammatory cytokines such as IL 1, IL-6 or TNF- $\alpha$, which in turn activate inducible nitric oxide synthase (iNOS). Nitric oxide (NO) is derived from the iNOS pathway potentiates the 


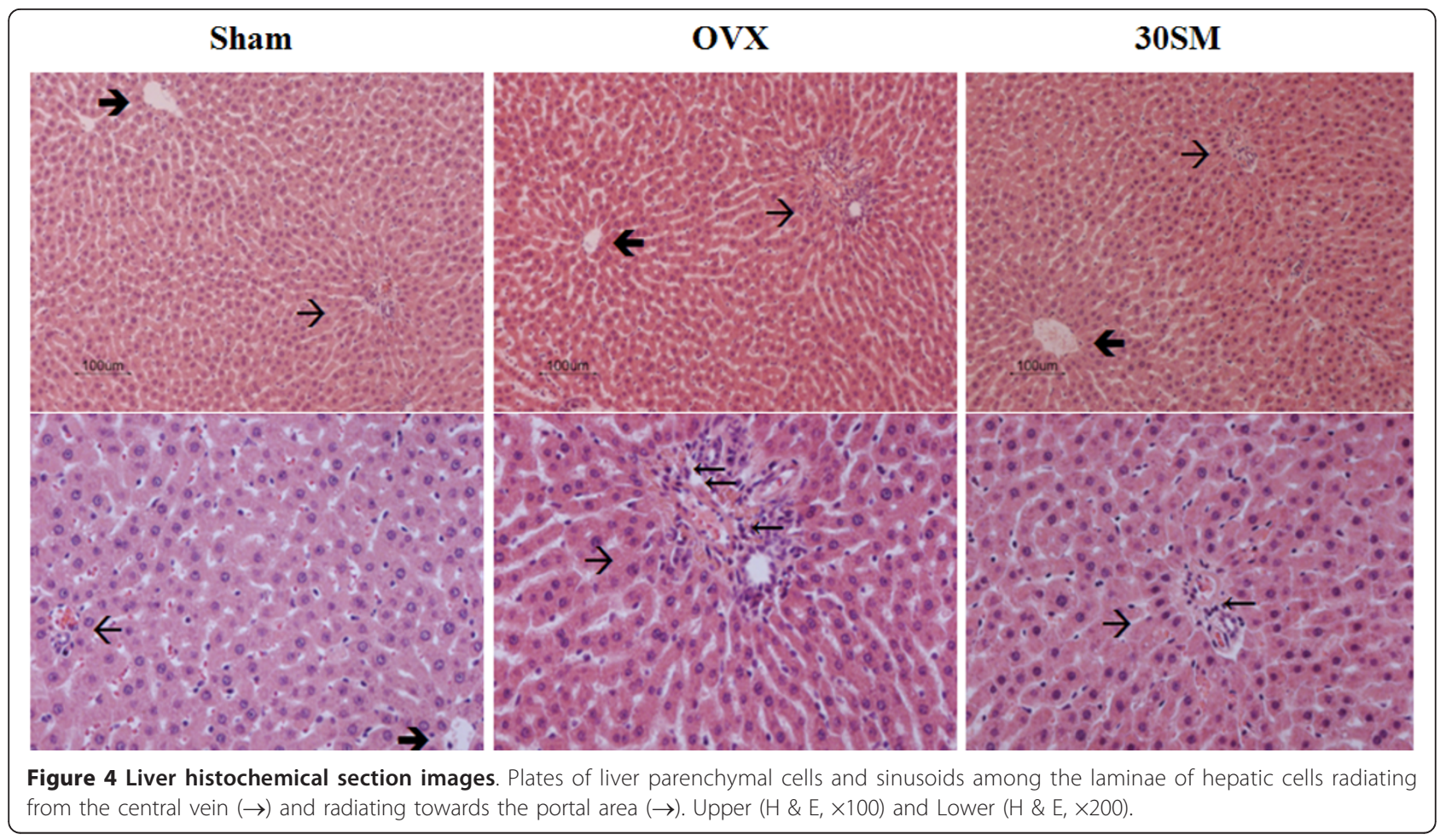

inflammatory cytokine-associated bone loss [25]. These studies give a possible explanation for the detected significant increase in the plasma nitrates level present in the OVX-rats in our study. Malondialdehyde (MDA) was also significantly increased in the OVX rats indicating increased oxidative stress. In the current study, SM treatment regulated the production of $\mathrm{NO}$ and MDA, which are related with bone resorption. It has been demonstrated that free radicals intervene in bone resorption, promoting osteoclastic differentiation [26]. Considering that enhanced osteoclastic activity in OVX rats has been suggested to be responsible for increased ROS [27], the regulatory effect of SM on NO and MDA could be one of the anti-osteoporotic mechanisms of the natural product.

In this study, SM treatment also significantly attenuated the increase in bone osteoclast number and serum TRAP-5b but did not affect the increase in serum BALP and ALP or in osteocalcin and estradiol induced by OVX. Generally, in subjects with normal liver function, serum ALP is similar to BALP and reflects osteoblast function [28]. Together with osteocalcin, they are markers of bone formation, while TRAP-5b is a bone resorption marker [22]. In the serum biochemical assessment, OVX did not affect serum calcium and IP levels or PTH and calcitonin activity, but significantly increased free $\mathrm{T}_{4}$ activity compared with Sham rats (Table 4 ). Free $\mathrm{T}_{4}$ activity was significantly reduced in 30SM rats compared with OVX rats (also shown in Table 4). Thyroid hormones play an important role in bone remodeling [29]. Histomorphometric studies have shown that thyroid hormones stimulate osteoblastic and osteoclastic activities in cortical and trabecular bone [30]. Thyrotoxicosis is associated with increased bone turnover, which can lead to a resorption rate that exceeds the formation rate, thus resulting in bone loss [31]. Considering that an increased rate of bone turnover was observed in subjects loaded with suppressive doses of $\mathrm{T}_{4}$, the inhibition of the increase of $\mathrm{T}_{4}$ levels by SM further suggests that SM has a regulatory effect on bone turnover. Increases in bone turnover have been reported in the perimenopausal period in humans probably due to estrogen deficiency [32]. Consistently, estradiol decrease was observed in OVX rats (Table 4). The reduced estradiol was not recovered by SM treatment. But with the data about estrogen, we could not

Table 3 The number of infiltrated mononuclear cells in the portal area of liver/10 HPF $(\times 200)$.

\begin{tabular}{ccccccc}
\hline Parameters & $\begin{array}{c}\text { Sham } \\
\mathbf{n}=\mathbf{7}\end{array}$ & $\begin{array}{c}\text { OVX } \\
\mathbf{n = 7}\end{array}$ & $\begin{array}{c}\text { 1SM } \\
\mathbf{n = 7}\end{array}$ & $\begin{array}{c}\text { 3SM } \\
\mathbf{n}=\mathbf{7}\end{array}$ & $\begin{array}{c}\text { 10SM } \\
\mathbf{n = 7}\end{array}$ & $\begin{array}{c}30 \text { SM } \\
\mathbf{n}=\mathbf{7}\end{array}$ \\
\hline Infiltrated Mononuclear cellular NO/10 HPF & $1.57 \pm 1.27$ & $12.00 \pm 2.40^{*}$ & $12.00 \pm 2.39$ & $11.50 \pm 2.20$ & $10.38 \pm 2.26$ & $8.50 \pm 2.34$ \\
\hline
\end{tabular}

Note: Results are presented as the means \pm SD. ${ }^{*} p<0.001$ vs. Sham group; ${ }^{\#} p<0.05$ vs. OVX group. Rat groups as defined above (N=7/each group). 


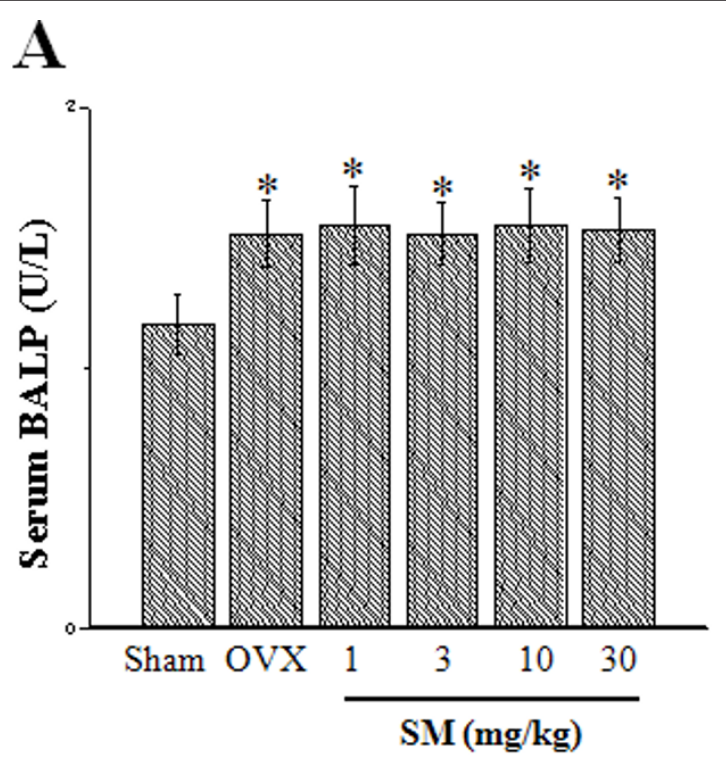

B
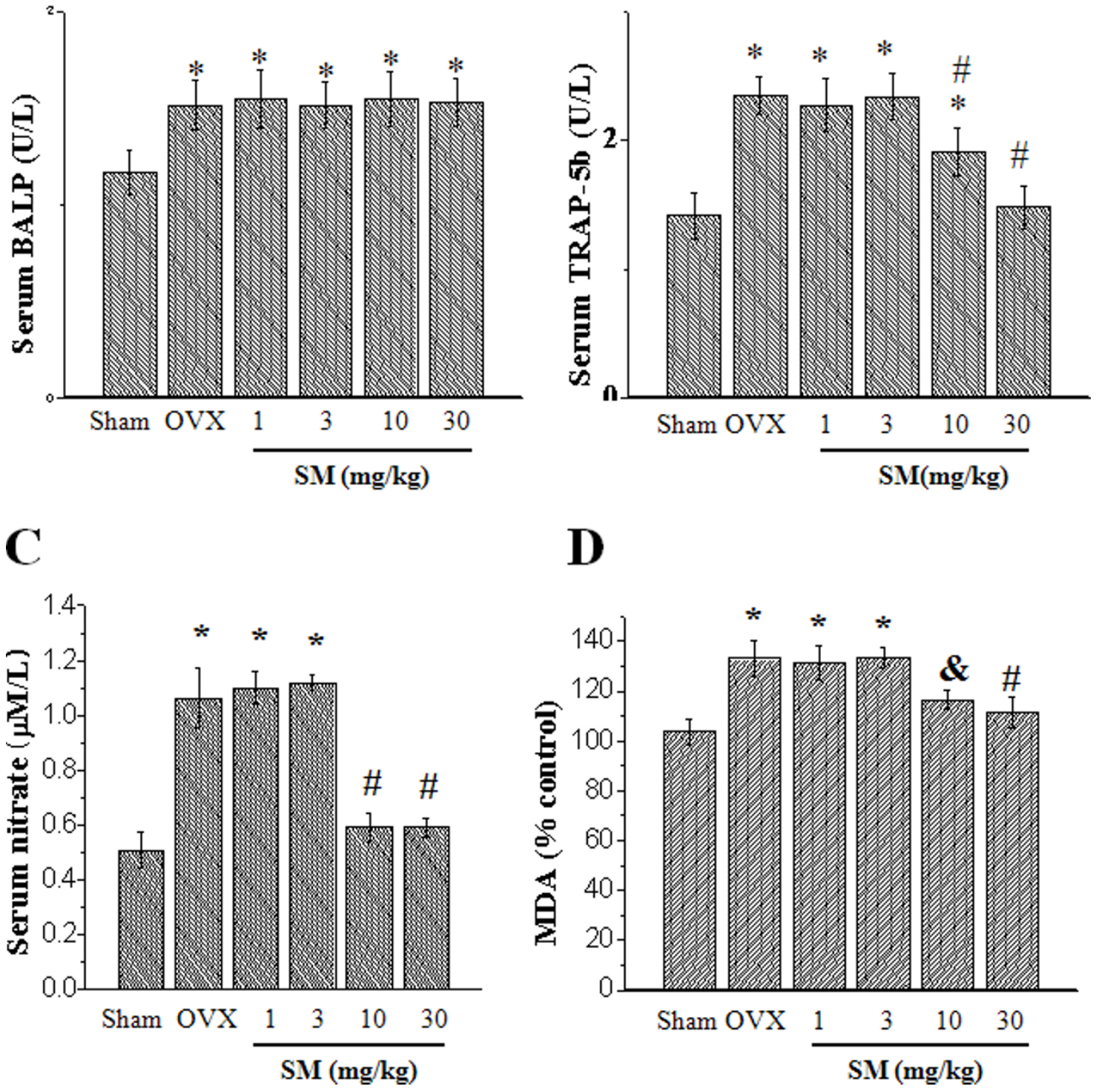

Figure 5 Serum bone turnover marker levels. Serum BALP activity (A), seru m TRAP-5b activity (B), Serum MDA levels (C) and serum nitrate levels (D) were measured as described in Materials and Methods. Note: (A) to (B) ${ }^{*} p<0.05$ vs Sham rats; ${ }^{*} p<0.05$ vs OVX rats. (C) ${ }^{*} p<0.001$ vs Sham group; ${ }^{\#} p<0.001$ vs OVX group. (D) ${ }^{*} p<0.001$ vs Sham rats; ${ }^{*} p<0.001$ vs OVX rats; ${ }^{*} p<0.01$ vs OVX rats.

Table 4 Serum biochemical parameters.

\begin{tabular}{ccccccc}
\hline Parameters & $\begin{array}{c}\text { Sham } \\
\mathbf{n}=\mathbf{1 0}\end{array}$ & $\begin{array}{c}\text { OVX } \\
\mathbf{n}=\mathbf{1 0}\end{array}$ & $\begin{array}{c}\text { SM1 } \\
\mathbf{n}=\mathbf{7}\end{array}$ & $\begin{array}{c}\text { SM3 } \\
\mathbf{n}=\mathbf{8}\end{array}$ & $\begin{array}{c}\text { SM10 } \\
\mathbf{n}=\mathbf{1 0}\end{array}$ & $\begin{array}{c}\text { SM30 } \\
\mathbf{n}=\mathbf{9}\end{array}$ \\
\hline ALP $(\mathbf{U} / \mathbf{L})$ & $32.57 \pm 8.28$ & $51.29 \pm 7.78^{\&}$ & $47.71 \pm 5.38$ & $51.57 \pm 3.74$ & $51.14 \pm 8.80$ & $47.57 \pm 7.00$ \\
Estradiol (pg/mL) & $16.09 \pm 3.38$ & $9.29 \pm 2.51^{*}$ & $10.27 \pm 3.18$ & $8.10 \pm 3.03$ & $9.07 \pm 2.60$ & $10.10 \pm 3.23$ \\
Free T3 (pg/mL) & $3.84 \pm 0.50$ & $3.83 \pm 0.31$ & $3.97 \pm 0.31$ & $3.91 \pm 0.23$ & $3.79 \pm 0.41$ & $3.74 \pm 0.23$ \\
Free T4 (ng/dL) & $1.90 \pm 0.47$ & $3.04 \pm 0.25^{*}$ & $3.07 \pm 0.30$ & $2.89 \pm 0.31$ & $2.73 \pm 0.23$ & $2.00 \pm 0.51 \%$ \\
Osteocalcin (ng/mL) & $2.40 \pm 0.28$ & $3.66 \pm 0.48^{*}$ & $3.40 \pm 0.57$ & $3.63 \pm 0.44$ & $3.44 \pm 0.43$ & $3.47 \pm 0.42$ \\
Calcium (mg/dL) & $9.30 \pm 0.41$ & $9.46 \pm 0.29$ & $9.63 \pm 0.21$ & $9.78 \pm 0.46$ & $9.40 \pm 0.27$ & $9.70 \pm 0.51$ \\
IP (mg/dL) & $6.21 \pm 0.80$ & $6.24 \pm 0.65$ & $6.60 \pm 0.57$ & $6.50 \pm 0.72$ & $6.17 \pm 0.53$ & $6.49 \pm 0.79$ \\
\hline
\end{tabular}

Note: T3, triiodothyronine; T4, thyroxine, IP, inorganic phosphorus. Results are presented as the means \pm SD. ${ }^{*} p<0.001$ vs. Sham group; ${ }^{\#} p<0.01$ vs. Sham group; $\&_{p}<0.05$ vs. Sham group; ${ }^{\%} p<0.001$ vs. OVX group. Rat groups as defined above ( $=6 /$ each group). 
determine whether SM has hormone-like effect or not. Although we did not clarify the characteristics of SM about hormone-like effect, we are suggesting that SM prevents trabecular bone loss by modulating osteoclast activity including decreasing osteoclast number/by decreasing osteoclast maturation, resulting in the regulation of bone turnover rate rather than by deceasing estrogen level.

The pharmacokinetics studies of these active components of SM in animals showed that they are absorbed orally and randomized clinical trials and clinical experiences indicate that the SM products are safe with a low side-effect profile [5]. Therefore, SM is a promising osteoporosis therapeutic agent candidate, although the specific mechanism of the anti-osteoporotic effect of SM needs to be clarified.

\section{Conclusions}

The preventive effect of SM against osteoporosis was presumably due to its anti-oxidative stress partly via modulation of osteoclast maturation and number. In current study, SM has been suggested to be a promising osteoporosis therapeutic natural product.

\section{Additional material}

Additional file 1: Chromatogram of constituents from SM extracts by HPLC analysis. The chromatogram shows peaks about Tanshinone $\| A$ and cryptotanshinone. The retention time for cryptotanshinone and tanshinone IIA was 14.8 and $21.6 \mathrm{~min}$. The content of tanshinone IIA and cryptotanshinone in Salvia Miltiorrhiza was $106.56 \mu \mathrm{mg} / 10 \mathrm{mg}(1.07 \%)$ and $109.655 \mu \mathrm{g} / 10 \mathrm{mg}(1.10 \%)$.

Additional file 2: HPLC analysis methods for measurement of the standards of Tanshinone IIA and Cryptotanshinone. The Additional file shows HPLC analysis method for the standard chemicals "Tanshinone IIA and Cryptotanshinone".

Additional file 3: Changes in body weight growth in rats. The additional file shows the rat's body weights from the second week after OVX (including OVX and all drug administration groups).

Additional file 4: Coronal image of proximal-medial tibia taken ex vivo by $\mu$-CT. The additional file shows conditions for $\mu$-CT (references Set distal growth plate as reference level and $8 \mathrm{~mm}$ distal from distal growth plate as cortical area analysis cut level and the related transaxial image of tibial diaphysis).

\section{Abbreviations}

ALP: alkaline phosphatase; aBMD: bone mineral density measured by DEXA; $B M D$ : bone mineral density measured by micro- $C T$; $a B M C$ : bone minera content measured by DEXA; BV/TV: bone volume fraction; BALP: bonespecific alkaline phosphatase; BTM: bone turnover marker; DEXA: dual-energy X-ray absorptiometry; ELISA: enzyme-linked immunosorbent assay; HPLC: high performance liquid chromatography; IL: interleukin; iNOS: inducible nitric oxide synthase; IP: inorganic phosphorus; M-CSF: macrophage colony stimulating factor; MDA: malondialdehyde; $\mu$-CT: microcomputerized tomography; NO: nitric oxide; NTX: amino-terminal region of Telopeptides of type I collagen; Oc.N: osteoclast number; OVX: ovariectomy or ovariectomized; PTH: parathyroid hormone; ROS: reactive oxygen species; SD: standard deviation; SMI: structure model index; T3: triiodothyronine; T4: thyroxine; Tb.N: trabecular number; Tb.Pf: trabecular bone pattern factor; Tb. Sp: trabecular separation; Tb.Th: trabecular thickness; TNF-a: tumor necrosis factor a; TRAP: tartrate-resistant acid phosphatase; TRAP-5b: type 5 tartrate resistant acid phosphatase band

\section{Acknowledgements}

This work was supported by the National Research Foundation Grants (20100029477, 2009-0087202) and was partly supported by Korea Research Foundation (KRF-2008-313-E00540) and by Korea Science and Engineering Foundation Grants (R01-2007-000-20275-0). We appreciate YJ Chang for technical assistance about $\mu-C T$ (Basic Research Institute, Jeonju, Korea).

\section{Author details}

${ }^{1}$ Department of Pharmacology and Institute of Cardiovascular Research School of Medicine, Chonbuk National University, Jeonju, Chonbuk, South Korea. ${ }^{2}$ Department of Dental Pharmacology and Wonkwang Dental Research Institute, School of Dentistry, Wonkwang University, Iksan, Chonbuk, South Korea.

\section{Authors' contributions}

YC mainly performed the animal experiment, analyzed the data and wrote a draft. AM did quantitative analysis of danshen. SC partially wrote a draft. BB, $G L, B L$ and DK supported the animal experiment, especially for feeding and establishing osteoporosis model. HK and HC supervised the project and wrote the final paper. All authors read and approved the final manuscript.

\section{Competing interests}

We declare that we have no competing interests. We also declare that we have no financial and non-financial competing interests.

Received: 24 July 2011 Accepted: 28 November 2011 Published: 28 November 2011

\section{References}

1. Anon.: Osteoporosis prevention, diagnosis, and therapy. Jama 2001, 285:785-795

2. Vassilopoulou-Sellin R: Breast cancer and hormonal replacement therapy. Ann N Y Acad Sci 2003, 997:341-350

3. Wang $X$, Morris-Natschke SL, Lee KH: New developments in the chemistry and biology of the bioactive constituents of Tanshen. Med Res Rev 2007, 27:133-148.

4. Han JY, Fan JY, Horie $Y$, Miura S, Cui DH, Ishii H, Hibi T, Tsuneki H, Kimura I: Ameliorating effects of compounds derived from Salvia miltiorrhiza root extract on microcirculatory disturbance and target organ injury by ischemia and reperfusion. Pharmacol Ther 2008, 117:280-295.

5. Zhou L, Zuo Z, Chow MS: Danshen: an overview of its chemistry, pharmacology, pharmacokinetics, and clinical use. J Clin Pharmacol 2005, 45:1345-1359.

6. Zhu YZ, Huang SH, Tan BK, Sun J, Whiteman M, Zhu YC: Antioxidants in Chinese herbal medicines: a biochemical perspective. Nat Prod Rep 2004, 21:478-489.

7. Kim HH, Kim JH, Kwak HB, Huang H, Han SH, Ha H, Lee SW, Woo ER, Lee $\mathrm{ZH}$ : Inhibition of osteoclast differentiation and bone resorption by tanshinone IIA isolated from Salvia miltiorrhiza Bunge. Biochem Pharmacol 2004, 67:1647-1656.

8. Zhang Y, Akao T, Nakamura N, Duan CL, Hattori M, Yang XW, Liu JX: Extremely low bioavailability of magnesium lithospermate $B$, an active component from Salvia miltiorrhiza in rat. Planta Med 2004, 70:138-142.

9. Lay IS, Chiu JH, Shiao MS, Lui WY, Wu CW: Crude extract of Salvia miltiorrhiza and salvianolic acid B enhance in vitro angiogenesis in murine SVR endothelial cell line. Planta Med 2003, 69:26-32.

10. Wong RW, Rabie AB: Effect of Salvia miltiorrhiza extract on bone formation. J Biomed Mater Res A 2008, 85:506-512.

11. Ding Y, Soma S, Takano-Yamamoto T, Matsumoto S, Sakuda M: Effects of salvia miltiorrhiza bunge (SMB) on MC3T3-E1 cells. J Osaka Univ Dent Sch 1995, 35:21-27.

12. Chae HJ, Chae SW, Yun DH, Keum KS, Yoo SK, Kim HR: Prevention of bone loss in ovariectomized rats: the effect of Salvia miltiorrhiza extracts. Immunopharmacol Immunotoxicol 2004, 26:135-144.

13. Brouwers JE, Lambers FM, Gasser JA, van Rietbergen B, Huiskes R: Bone degeneration and recovery after early and late bisphosphonate treatment of ovariectomized wistar rats assessed by in vivo microcomputed tomography. Calcif Tissue Int 2008, 82:202-211. 
14. Jiang SD, Shen C, Jiang LS, Dai LY: Differences of bone mass and bone structure in osteopenic rat models caused by spinal cord injury and ovariectomy. Osteoporos Int 2007, 18:743-750.

15. Kim DS, Han SH, Lee YC, Park SJ, Yoo WH, Kim HR, Chae HJ: The study of the combination of Slavia Miltiorrhiza and Kitosan on osteoporosis in ovariectomized rats. Kor J Clin Pharm 2009, 19(2):75-80.

16. Jarvinen $T L$, Sievanen $H$, Kannus $P$, Jarvinen M: Dual-energy X-ray absorptiometry in predicting mechanical characteristics of rat femur. Bone 1998, 22:551-558.

17. Gothe S, Wang Z, Ng L, Kindblom JM, Barros AC, Ohlsson C, Vennström B, Forrest $D$ : Mice devoid of all known thyroid hormone receptors are viable but exhibit disorders of the pituitary-thyroid axis, growth, and bone maturation. Genes Dev 1999, 13:1329-1341.

18. Kannus P, Jarvinen TL, Sievanen H, Kvist M, Rauhaniemi J, Maunu VM, Hurme $T$, Jozsa $L$, Järvinen $M$ : Effects of immobilization, three forms of remobilization, and subsequent deconditioning on bone mineral content and density in rat femora. J Bone Miner Res 1996, 11:1339-1346.

19. Kastl S, Sommer T, Klein P, Hohenberger W, Engelke K: Accuracy and precision of bone mineral density and bone mineral content in excised rat humeri using fan beam dual-energy X-ray absorptiometry. Bone 2002, 30:243-246.

20. Draper HH, Hadley M: Malondialdehyde determination as index of lipid peroxidation. Methods Enzymol 1990, 186:421-431.

21. Bories PN, Bories C: Nitrate determination in biological fluids by an enzymatic one-step assay with nitrate reductase. Clin Chem 1995, 41:904-907.

22. Cremers S, Garnero P: Biochemical markers of bone turnover in the clinical development of drugs for osteoporosis and metastatic bone disease: potential uses and pitfalls. Drugs 2006, 66:2031-2058.

23. Halleen JM, Tiitinen SL, Ylipahkala H, Fagerlund KM, Vaananen HK: Tartrateresistant acid phosphatase $5 b$ (TRACP $5 b$ ) as a marker of bone resorption. Clin Lab 2006, 52:499-509.

24. Kireev RA, Tresguerres AC, Garcia C, Borras C, Ariznavarreta C, Vara E, Vina J, Tresguerres JA: Hormonal regulation of pro-inflammatory and lipid peroxidation processes in liver of old ovariectomized female rats. Biogerontology 2010, 11:229-243.

25. Van't Hof RJ, Ralston SH: Nitric oxide and bone. Immunology 2001, 103:255 261.

26. Sánchez-Rodríguez MA, Ruiz-Ramos M, Correa-Muñoz E, MendozaNúñez VM: Oxidative stress as a risk factor for osteoporosis in elderly Mexicans as characterized by antioxidant enzymes. BMC Musculoskelet Disord 2007, 19(8):124.

27. Koh JM, Lee YS, Kim YS, Kim DJ, Kim HH, Park JY, Lee KU, Kim GS: Homocysteine enhances bone resorption by stimulation of osteoclast formation and activity through increased intracellular ROS generation. J Bone Miner Res 2006, 21(7):1003-11.

28. Farley JR, Chesnut CH, Baylink DJ: Improved method for quantitative determination in serum of alkaline phosphatase of skeletal origin. Clin Chem 1981, 27:2002-2007.

29. Mosekilde L, Eriksen EF, Charles P: Effects of thyroid hormones on bone and mineral metabolism. Endocrinol Metab Clin North Am 1990, 19:35-63.

30. Mosekilde L, Melsen F, Bagger JP, Myhre-Jensen O, Schwartz Sorensen N: Bone changes in hyperthyroidism: interrelationships between bone morphometry, thyroid function and calcium-phosphorus metabolism. Acta Endocrinol (Copenh) 1977, 85:515-525.

31. Madsen JE, Aune AK, Falch JA, Hukkanen M, Konttinen YT, Santavirta S, Nordsletten L: Neural involvement in post-traumatic osteopenia: an experimental study in the rat. Bone 1996, 18:411-416.

32. Nilas $L$, Christiansen C: Rates of bone loss in normal women: evidence of accelerated trabecular bone loss after the menopause. Eur I Clin Invest $1988,18: 529-534$.

\section{Pre-publication history}

The pre-publication history for this paper can be accessed here: http://www.biomedcentral.com/1472-6882/11/120/prepub

doi:10.1186/1472-6882-11-120

Cite this article as: Cui et al:: Characterization of Salvia Miltiorrhizo ethanol extract as an anti-osteoporotic agent. BMC Complementary and Alternative Medicine 2011 11:120.

\section{Submit your next manuscript to BioMed Central and take full advantage of:}

- Convenient online submission

- Thorough peer review

- No space constraints or color figure charges

- Immediate publication on acceptance

- Inclusion in PubMed, CAS, Scopus and Google Scholar

- Research which is freely available for redistribution

Submit your manuscript at www.biomedcentral.com/submit 